\title{
The new EU regulation on in vitro diagnostics: potential issues at the interface of medicines and companion diagnostics
}

\begin{abstract}
The new European regulation for in vitro diagnostics (IVD) divides the certification of IVD including companion diagnostics (CDx) by notified bodies (NB) from the market authorization of medicines. With the new regulation, $\mathrm{CDx}$ will require conformity assessment which is expected to include clinical evidence by NB. This is a significant change from the current situation: until now most IVD have been certified based on their manufacturers' assessment. For one medicine assessed by the EMA, certification of several different CDx by different NB is possible. As the benefit-risk balance of the medicine may depend on the performance (e.g., sensitivity and specificity) of its CDx, a close cooperation of EMA and NB will be necessary. The availability of detailed information on $\mathrm{CDx}$ used in the pivotal clinical trials for the medicine's authorization will become crucial for the assessment of alternative or competing CDx.
\end{abstract}

First draft submitted: 20 August 2016; Accepted for publication: 6 September 2016; Published online: 23 September 2016

Keywords: benefit-risk balance $\bullet$ CE mark $\bullet$ certification $\bullet$ companion diagnostics - conformity $\bullet$ in vitro diagnostics $\bullet$ marketing authorization $\bullet$ medical devices $\bullet$ notified bodies $\bullet$ regulation

\section{The regulation of the European} Parliament \& of the Council on in vitro diagnostic medical devices Research and development, regulatory authoriza tion and decisions on price and reimbursement of innovative medicines evolve and adapt to changing conditions and requirements $[1,2]$. There is an obvious trend leading away from the blockbuster principle toward a personalized precision medicine. This development is driven by an increasing stratification of patients by new diagnostic options. At the same time, the broad and sustainable availability of accurate and precise diagnostic tests (biomarkers) become crucial factors for the success of the innovative targeted therapies [3]. The close link between diagnostic test (companion diagnostics [CDx], in vitro diagnostics [IVD] or medical device) and medicine (medicinal product or pharmaceutical) and the importance of a CDx for the safety and efficacy of targeted therapies strongly suggest that comparable regulatory standards and requirements should apply for CDx and medicines [4]. Accordingly, regulatory frameworks have been created, for example, in the USA [5] and Japan [6]. At variance, until now, CDx have not been defined in the European legislation. According to the (old) IVD directive, the notified bodies (NB) have been responsible for all IVD and for most of them the conformity assessment by their manufacturer has been sufficient. Now the proposal for a regulation of the European Parliament and of the Council on IVD medical devices is progressing in the legislative procedure [7]. This regulation will for the first time in EU legislation specifically address CDx. Importantly, $\mathrm{CDx}$ will be classified as high individual risk or moderate public risk (category $\mathrm{C}$ ) requiring a conformity assessment not only by their
Harald Enzmann ${ }^{*, 1}$, Ralf Meyer ${ }^{1} \&$ Karl Broich ${ }^{1}$ 'Bundesinstitut für Arzneimittel \& Medizinprodukte, Bonn, Germany *Author for correspondence: Tel.: + 49228993073315 Harald.enzmann@bfarm.de
Future $\because \%$ Medicine part of 
manufacturer but also by NB and the importance of the clinical performance and of clinical evidence for the conformity are emphasized [8].

Although the proposed regulation makes the assessment of CDx more similar to the assessment of medicines than it has been in the EU in the past, it upholds the split responsibilities for medicinal products and medical devices (including IVD and CDx). There is a far-reaching mandate for the EMA for medicinal products (medicines). Since 2004, innovative medicines have been authorized for the European market virtually exclusively by EMA's centralized procedure. Responsibility for medical devices (including CDx) remains with the multitude of $\mathrm{NB}$, even when the use of $\mathrm{CDx}$ and the treatment with a medicine are tightly linked. It is at the discretion of the NB to consult either EMA or national competent authorities (NCA) in the member states on the assessment of CDx. There is, however, no need for a consensus. EMA or NCA cannot veto the decision of the $\mathrm{NB}$ on a CDx but they will need to consider a potential effect of the CDx on the benefit-risk balance of the corresponding medicine. Similarly, health technology assessment (HTA) bodies may want to include in their evaluations the effects of a CDx on cost-effectiveness of a treatment.

\section{The proposed definition of CDx}

A plethora of IVD (including the whole range of clinical chemistry) has long contributed to medical diagnosis, supplementing patients' medical history, symptoms, clinical findings and other invasive (e.g., endoscopy) or noninvasive (e.g., imaging) diagnostic procedures. A physician's treatment decision for an individual patient will usually integrate the whole available information (including patient's preference and consent). The use of innovative precision medicines may depend to a much greater extent on the result of a single IVD. This is reflected in the proposed definition of the CDx as 'essential for the safe and effective use of a corresponding medicinal product'.

A CDx will usually look at a specific property, frequently a molecular change, which is crucial for the efficacy or safety of the precision medicine. This property may be inherent in patients or a characteristic of the pathogen (i.e., the infectious agent or tumor). A CDx can be related to the mode of action of the targeted therapy and assess the sensitivity of the pathogen (e.g., the presence of receptors on tumor cells); or a CDx can focus on particularities in the metabolism of patients which may make them unusually vulnerable for the targeted therapies' adverse effects.

The demonstration of specific properties of the pathogen has long been accepted in clinical practice as a prerequisite for specific therapy. Antibiotic treatment will depend on the bacteria's sensitivity, in other words, the results of the antibiogram. The decision for the hormone therapy of breast cancer will depend on the cancer cells' receptor status. But there is an interesting difference between these two examples: initiation of the antibiotic therapy is not only acceptable but also mostly necessary before the results of the antibiogram become available; initiation of anti-estrogen therapy of breast cancer without knowledge on the tumor cells' receptor is not. If the demonstration or exclusion of a specific property of the pathogen or patient has been made mandatory in the summary of product characteristics ( $\mathrm{SmPC}$ ) of the medicine, any prescription without would constitute an off-label use - with all its legal (e.g., patient's consent) and economic consequences (e.g., exclusion from reimbursement).

Similar to the properties of pathogens (e.g., bacteria, viruses and tumor cells), specific characteristics of individual patients (possibly completely unrelated to the disease) may be evaluated by IVD and may become crucial for treatment decisions. Important examples are QT interval prolongation and cytochrome polymorphisms, which may exclude patients from treatment with some substances. As both QT interval prolongation and cytochrome polymorphisms are patient specific but independent of a specific disease, they are relevant not only for one specific pharmacologically active substance but also for several medicines. Once a certain phenotype, for example, lack of CYP2D6 has been established, it will of course be relevant for various medicines metabolized by CYP2D6, without the need (or even the justification) to repeat the determination, even years later when a different medicine is considered for the treatment of a different medical condition.

Regarding tests for common cytochrome polymorphisms as CDx may seem counterintuitive as the result will be important for several medicines used for the treatment of unrelated diseases. According to the currently proposed definition, however, any test that can be used to determine, for example, the CYP2D6 status will become a CDx as the CYP2D6 status of patients has been explicitly included in the indication of Cerdelga ${ }^{\circledR}$ (eliglustat) and thus is obviously essential for the safe and effective use of this medicine [9].

At variance with previous draft versions of the regulation the latest version gave up the definition of a $\mathrm{CDx}$ as "a device specifically intended to select patients ... for a targeted therapy" and widened the definition to "essential for the safe and effective use of a corresponding medicinal product to: - identify, before and/or during treatment, patients who are most likely to benefit ... or - patients likely to be at increased risk for serious adverse reactions as a result of treatment..." [10]. There- 
fore, it does no longer matter whether or not the test is intended to be used for the selection of patients with Gaucher disease type 1 for treatment with Cerdelga. Many products previously simply regarded as IVD will fall in this broad definition of a CDx, including tests for cytochrome polymorphisms. It may seem inconsistent that an exemption has been proposed for devices for therapeutic drug monitoring as the associated risks are similar to the risks of cytochrome polymorphisms; both therapeutic drug monitoring and the determination of cytochrome polymorphism are used to avoid exceedingly high and toxic or exceedingly low and ineffective plasma concentrations of the active substance or its metabolites.

There may be some room for interpretation of the exact meaning of 'essential' in the proposed definition of a CDx. To what extent should an IVD take a gatekeeper role [4] for a pharmacological treatment to be considered a CDx? The use of an IVD procedure (not necessarily of a specific product, see below) may be mandatory for some medicinal products and only 'recommended' for others. Will a test be considered essential if it is optional? Will a test be essential if the 'wrong' result is unambiguously prohibitive for treatment but patients with unknown status may be treated anyway? Will a mandatory test be considered essential, if it contributes to the overall treatment decision without pre-empting this decision either way? In that case a positive result of the $\mathrm{CDx}$ would clearly favor treatment without outweighing other contraindications, whereas a negative result would indicate a less favorable situation but still allow a treatment based on a holistic assessment and a positive benefit-risk balance. An interpretation restricting the definition of CDx only to mandatory tests that are part of a medicine's indications or contraindications seems the most pragmatic approach.

With the proposed definition of a CDx, it may seem unnecessary that the product is specified in the SmPC; it is sufficient that the demonstration (or exclusion) of a crucial finding (e.g., a mutation) is mandatory for the use of the medicine and any product or method used for the demonstration (or exclusion) of this finding will have to fulfill the requirements for a class $\mathrm{C}$ medical device. It would be EMA's responsibility to be very precise in the wording of the SmPC defining which finding (e.g., mutation or receptor or enzyme polymorphism) is a prerequisite or is prohibitive for a treatment with a medicinal product. It would then be the responsibility of the NB to categorize any products that can be used to demonstrate or exclude this finding as CDx (risk class C). This is consistent with EMA's current practice describing findings rather than naming specific products in the SmPC but at variance with the US approach, which is focused on the product rather than the finding. US FDA assesses on product level both the medicine and the CDx status for the IVD.

The European regulators' role in the evaluation of IVD or CDx is ambiguous. The new regulation deliberately divides the responsibilities: medicinal products to the EMA and NCA and IVD together with medical devices to the NB (with a consultation of EMA and/or NCA for CDx). Clear as this separation of responsibility may seem regulators still need to consider the impact CDx may have on the benefit-risk balance of a medicine - particularly if a positive benefit-risk balance depends on the features of the CDx.

\section{The conformity assessment by NB \& the benefit-risk assessment by EMA}

For medical devices including IVD and CDx the certification process in the EU differs from the authorization of medicinal products. Innovative medicinal products receive a marketing authorization from the European Commission based on a positive benefitrisk assessment by the EMA. Medical devices including IVD may be sold in the EU with a CE mark after assessment and approval from a NB. For most devices or IVD in risk class $\mathrm{A}$ and $\mathrm{B}$ the certification will focus on the technical features and technical quality of the products. For products in risk class $\mathrm{D}$ and risk class $\mathrm{C}$ (e.g., $\mathrm{CDx}$ ) the proposed regulation puts much emphasis on the assessment of the clinical performance and clinical evidence for conformity. It will be important to distinguish the accuracy and precision of the laboratory method (e.g., the detection of specific receptor proteins or nucleic acid sequences in a tumor sample [11]) from its predictive value for clinical medicine (e.g., sensitivity and specificity for the efficacy of a medicine). An assay for the detection of presumably cardionoxious autoantibodies [12] may have a high accuracy for the detection of these antibodies and be useful to evaluate the true prevalence of the antibodies. To qualify as a CDx, its predictive value for efficacy or safety of therapies targeting the auto-antibodies needs to be shown in a second step. It may be challenging and sometimes impossible to use the relevant clinical outcome, for example, an increased survival time of oncology patients, for the demonstration of sensitivity and specificity for the efficacy of a treatment. Depending on the mode of action of the medicine appropriate surrogate parameters such as tumor response rate may be considered.

The consultation of institutions responsible for the regulation of medicines (EMA and NCA) during the certification process for a CDx will support the development of compatible and consistent decision cri- 
teria for medicines and devices. The availability of sufficient resources for the clinical assessment by the NB will become important as currently approved medical devices are not exempt from the increased requirements of the regulation. After the expected transitional period of 5 years they will need to be re-evaluated and re-approved. It may be of importance that the end of the transitional periods may coincide with Brexit becoming effective. The worst case scenario would be an unregulated termination of UK's EU membership, 2 years after invocation of Article 50 and after utterly failed exit negotiations. In that case the UK's NCA could no longer accredit the NB and without accreditation the UK-based NB may no longer certify devices and IVD, in other words, grant the CE mark. The NB in a post-Brexit EU would at the same time face the increased workload required by the regulation and would need to compensate for the loss of the contribution from UK-based NB. An appropriate mutual recognition agreement between the $\mathrm{EU}$ and the $\mathrm{UK}$, following the examples of NB in Norway, Switzerland, Turkey and Australia, may avoid this problem [13].

Regulators need to consider that the benefit-risk balance of a medicine may depend on the accuracy of the IVD used to select patients for or exclude patients from a treatment. Changes in precision and accuracy of the CDx and its sensitivity and specificity for the identification of the patients' eligible for the treatment equal a change of selection criteria. The positive benefit-risk balance in the pivotal studies may not remain the same when the selection criteria are altered by the use of different CDx post-approval. Any decrease in sensitivity and specificity of the IVD in comparison to the pivotal studies must be expected to increase the number of patients who are wrongly selected for or wrongly excluded from a treatment with a medicine. If a specific finding or a specific result of an IVD is a necessary condition for the treatment with the medicinal product, a decrease in sensitivity will deprive more patients than unavoidable (e.g., avoidable by the use of an IVD with higher sensitivity) from the benefits of the treatment. The benefit-risk balance for the medicine remains positive for the patients who will receive the treatment. Therefore, regulators who are responsible for the positive benefit-risk balance have no justification for decisive regulatory action; they may, preferably in consensus with the NB, inform about the differences in sensitivity of the IVD and its possible consequences for patients but have no legal grounds to modify the marketing authorization of the medicinal product. This may be different for a decrease in the IVD's specificity. In this case more patients will be exposed to the risks of the treatment without justification of these risks by a chance to benefit from the treatment, in other words, the number needed to treat will increase. Regulators will need to consider whether the positive benefit-risk balance may be jeopardized, and whether a minimum specificity of the IVD is required for a positive benefit-risk balance. If the use of an inferior IVD test with an unacceptable low specificity results in a negative benefit-risk balance of the medicinal product, regulators will need to implement measures to stop or prevent the IVD-driven misuse of an otherwise beneficial medicine.

\section{The common responsibility of NB, EMA \& HTA bodies}

NB will assess the technical features and technical qualities of a CDx, EMA will assess the benefit-risk balance of the medicine, both NB and EMA regulators will need to consider the impact of the $\mathrm{CDx}$ on the treatment of patients, in other words, potential changes in the benefit-risk balance of the medicine and HTA will look at the cost-effectiveness of the combination of $\mathrm{CDx}$ and medicine. As long as the benefit-risk balance for a treatment with a medicine remains positive, regulators can accept the parallel or alternative use of different diagnostic methods for the selection of patients. If the benefit of a treatment is outstanding, the benefit-risk balance will remain positive, even if patients are selected with an IVD test with inferior specificity. The increase in numbers needed to treat will not make the benefit-risk balance negative. This is why the SmPC for Herceptin (trastuzumab) lists several methods for the determination of HER2 status. The wide range of accepted methods includes HER2 gene amplification by fluorescence in situ hybridization or chromogenic in situ hybridization for breast cancer or silver-enhanced in situ hybridization for gastric cancer and "any other method that may be used for the assessment of HER2 protein or gene expression" under the condition that this method is "able to distinguish between moderate (congruent with 2+) and strong (congruent with 3+) overexpression of HER2" [14]. It may be presumed that these methods even when performed "by laboratories that provide adequate state-ofthe-art performance of validated method" will differ to some extent in sensitivity and specificity. Due to the impressive efficacy of Herceptin even a significant increase in the number needed to treat will not make Herceptin's benefit-risk balance negative. This does not mean that differences in the accuracy of IVD should be ignored. The deliberate preference for an IVD with an inferior accuracy would seem unjustified and certainly not in patients' interest. It will be the responsibility of the NB that certified the (inferior) CDx to prevent harm to patients. EMA's regulatory mandate is for the medicine, not the IVD. As long as 
the benefit-risk balance for the medicine remains positive when patients' selection for treatment is based on a CDx with inferior accuracy, EMA cannot limit the use of the medicine. Patients for whom the benefit-risk balance is still positive (even after selection by an inferior $\mathrm{CDx}$ ) cannot be excluded from treatment without doing more harm by depriving them of a potentially beneficial treatment.

At variance, the mere conclusion that the benefitrisk balance is positive cannot be sufficient for HTA. If the price of a medicine is linked to the extent of its benefit, a more exact quantification of the benefit with regard to size (e.g., increase in time of survival or without symptoms) and frequency (effective in how many of treated patients) will be required. It matters little whether these data are used to calculate the price of quality adjusted life years (e.g., as NICE does for England) or whether they are used in a categorical approach (using, e.g., the categories no, minor or considerable benefit) as preferred by the German federal joint committee. In any case HTA bodies will aim to distinguish between two possible scenarios. On the one hand, there is the high inclusion threshold scenario: setting the evaluation criteria for a high specificity (and a consequently relatively low sensitivity) will restrict treatment to a smaller number of patients who will have on average a higher benefit. Under these conditions the number of patients at risk for the adverse effects of the medicine without the benefit of an efficacious treatment is kept at a minimum, whereas the number of patients who may have benefited from the treatment but were (wrongly) excluded from these benefits will be higher. On the other hand, the low inclusion threshold scenario will offer the treatment to a greater number of patients, minimizing the number of patients wrongly excluded from the benefits of the treatment by a high sensitivity of the IVD. At the same time, the corresponding decreased specificity must be expected to result in a higher number of patients who will suffer the adverse effects without profiting from the efficacy of the medicine.

The prediction that $\mathrm{CDx}$ will at least short-term increase rather than decrease the overall costs [15] will sensitize HTA bodies to the fact that a benefit-derived price and overall costs may to a significant extent depend on the threshold for the selection of patients. The high inclusion threshold scenario means treatment of fewer patients with a higher benefit resulting in fewer sales of the medicine possibly at a higher price. This may be economically attractive particularly in oncology [16], where targeted therapeutics benefit only a minority of patients and high prices limit the room for further price increases. The low inclusion threshold scenario will allow the treatment of more patients but the average benefit will be lower (due to an increased number of nonresponders), presumably resulting in more sales of the medicine but justifying only a lower price.

\section{Effects of CDx on the benefit-risk balance $\&$ license status of medicines}

A CDx can obtain its certification for marketing in the EU, in other words, the CE mark, from any of the multiple NB. EMA or NCA may be invited to express their position but their consensus is not necessary. How an IVD is used to select patients for a treatment, in other words, the definition of a selection process or selection threshold in the SmPC of the medicine is to a considerable extent the decision of the marketing authorization holders. Regulators can only partly correct the possibly economically driven decisions. On the one hand, regulators may prevent a specificity that is unacceptably low. Including too many patients who will have little or no benefit from the treatment (while at risk for the adverse effects) may turn the benefit-risk balance of the medicine negative and justify the refusal of the marketing authorization. On the other hand, if the applicant focused on the (few) patients with the very best chances for pronounced efficacy and recommended or required the use of a CDx with an exceedingly high specificity and a correspondingly quite low sensitivity, regulators would certainly express their view and advice different cut-off criteria. They could not, however, refuse marketing authorization for a medicine with a CDx-dependent deliberately narrow indication. The benefit-risk balance for these possibly few, most suitable patients is positive after all. There would be neither an ethical justification nor legal grounds for denying these patients the benefit of this medicine, even if there were serious concerns that other patients who may be expected to benefit were unnecessarily excluded from the treatment. Only if this exceedingly narrow indication was overcompensated by significant off-label use, with evidence that the medicine was used in patients for whom the benefit-risk balance was negative, decisive regulatory action would be possible.

Differences in specificity will have the most impact in situations with high rareness of the required biomarker result. This is obviously relevant for orphan diseases and is becoming increasingly more important for rare genotype subgroups of common cancers. Under these circumstances efficacy of a medicine will frequently be predicted almost exclusively on the basis of the biomarker results, which will have an overwhelming influence on the treatment decision. This must be put in the context of the usually severe adverse effects of antineoplastic medicines including targeted therapies. For illustration of the importance of specificity we con- 
sider a crucial biomarker for a targeted therapy being predictive for efficacy, in other words, we presume that patients without this biomarker will not benefit from the treatment. We shall consider specificity only and assume perfect sensitivity. If a hypothetical biomarker indicated efficacy of the targeted therapy in one out of 500 patients, even a specificity of $99.8 \%$ would mean that for each patient correctly identified for treatment by a positive biomarker result, another patient would be treated based on a false-positive result. Under these conditions, half of the patients treated would suffer the adverse effects without a chance to benefit from the treatment. Depending on the expected benefits of an efficacious treatment and on the severity and frequency of the adverse effects this may be acceptable. Under the same conditions, but with a specificity of the biomarker test of $99 \%$, for each correctly treated patient five patients would be falsely identified as eligible for the treatment. This may not be acceptable, neither from patients' benefit-risk perception nor from payers' costeffectiveness perspective. A certification (CE mark) for such an IVD cannot be justified, even if its specificity was technically correctly described as $99 \%$. Such an increase in the number needed to treat may well turn the benefit-risk balance negative. Or it may significantly increase the costs if more patients (e.g., threetimes as many in this hypothetical example) were to receive an expensive treatment - without an increase in the number of patients who would actually benefit. This example clearly illustrates the importance of the assessment of the clinical performance of IVD and why the need for clinical evidence for the conformity is emphasized in the proposed regulation. EMA and NCA regulators have no or only limited and indirect responsibility for IVD, CDx and the cost-effectiveness of the use of medicines. What they do have is an explicit mandate to assure a positive benefit-risk balance of medicines marketed in the EU. As illustrated above, changes in a test's performance, for example, in accuracy, may drastically alter the numbers needed to treat. Particularly in case of medicines with severe adverse effects it is quite conceivable that this may turn the benefit-risk balance negative. The introduction of appropriate safeguards against unfavorable combinations of medicine and CDx seem warranted. Transparent information and cooperation of EMA, NB and HTA bodies will be essential, with HTA bodies and payers possibly becoming the goal-keepers against combinations of medicines and CDx, each on their own approved by EMA or NB but useless or even harmful in a specific combination.

\section{Conclusion}

As the regulation separates responsibilities for medicines and IVD including CDx, both the EMA and NB will have crucial roles in market access decisions for precision medicines and the corresponding CDx. For one medicine assessed by the EMA, certification of several different $\mathrm{CDx}$ by different $\mathrm{NB}$ is possible and the specific products need not be explicitly named in the $\mathrm{SmPC}$ of the medicine. Differences in the performance of competing CDx products may change the selection of patients for the treatment and may thus alter the benefit-risk balance of the medicine. It is all stakeholders' and most importantly patients' interest that the EMA provide detailed information (accuracy, precision, sensitivity and specificity as appropriate) on the CDx used in the pivotal trials that were the basis for the EMA's positive benefit-risk assessment. Only then other decision makers, for example HTA bodies, payers, physicians and patients can build on EMA's assessment and evaluate the suitability of alternate $\mathrm{CDx}$ according to their own criteria. Detailed and objective information on CDx and medicines by NB and EMA will be more useful for informed decisions of downstream stakeholders than a rigid regulatory coupling of a specific medicinal product to one specific CDx and vice versa.

Overall, the new European regulation on IVD will assure adequate standards for CDx, is open for competing products and leaves room for decisions of other stakeholders.

\section{Future perspective}

The regulation of the European Parliament and of the Council on IVD medical devices will for the first time in EU legislation specifically address CDx. The regulation maintains clearly separated responsibilities for medicines and IVD including CDx. For market access, innovative medicines need marketing authorization based on a positive benefit-risk assessment by the EMA, whereas IVD are certified, in other words, obtain the CE mark, by any of numerous NB. According to the proposed definition, any IVD used to demonstrate or exclude findings that are a crucial part of the indications or contraindications in a medicine's marketing authorization will be regarded as CDx. The specific CDx products that enable the evaluation of these findings need not be explicitly named in the $\mathrm{SmPC}$ of a medicine. CDx are classified as high individual risk or moderate public risk (category $\mathrm{C}$ ) requiring a conformity assessment not only by their manufacturer but also by NB, including an evaluation of the clinical performance and an assessment of the clinical evidence for the conformity of the CDx.

Currently approved medical devices are not exempt from the regulation. They will need to be re-evaluated and re-approved according to the new requirements, probably after a transitional period of five years. The assessment of the clinical evidence by the NB will be a 
challenge for the sufficient availability of expertise. The consultation of EMA and NCA in CDx procedures may provide some support.

Differences in the performance of competing CDx products may change the selection of patients for the treatment and may alter the benefit-risk balance of the medicine. The EMA will be required to provide detailed information on $\mathrm{CDx}$ used in the trials that were the basis for the marketing authorization. Exceeding the mere description of the characteristics of the CDx used in the pivotal studies, EMA could define in their initial assessment of a new medicine the minimum requirements for the performance of $\mathrm{CDx}$. Although it is certainly difficult to set exact cut-offs for a test's acceptable and not acceptable performance, a scientific discussion what extent of changes in specific- ity and sensitivity may critically alter the benefit-risk balance would support other stakeholders' assessment of the advantages and limitations of different $\mathrm{CDx}$ products used in conjunction with a medicine.

If EMA and NB work together as intended, the new regulation will successfully address both the need to assure the safe use of CDx and the need to support the development of more and better CDx. Based on EMA's assessment how different performance characteristics of $C D x$ are expected to change the benefit-risk balance of the medicine, all NB will refuse the certification of products that fail to meet these minimum requirements and thus set a European standard. Once a product meets this standard, other stakeholders may make their own decision based on their own criteria and the information provided by NB and EMA on the charac-

\section{Executive summary}

EU legislation will for the first time specifically address companion diagnostics in the regulation on in vitro diagnostic medical devices

- The regulation of the European Parliament and of the Council is progressing in the legislative procedure.

- It will profoundly change the certification of companion diagnostics (CDx) and will impact the use of and possibly the regulation of medicines as well.

The regulation uses a broad definition of CDx

- At variance with previous draft versions of the regulation the latest version gave up the definition of a CDx based on its intended use and widened it to "essential for the safe and effective use of a corresponding medicinal product."

- Widely used test, for example, for cytochrome polymorphisms, will fall under this definition.

The conformity assessment by notified bodies remains different from the benefit-risk assessment by EMA

- The regulation maintains the separation between medicines' marketing authorization based on a positive benefit-risk assessment by the EMA and certification of CDx by any of numerous notified bodies (NB).

- CDx will be considered risk class $C$ products requiring clinical evidence of conformity for assessment by NB. This is a significant change for in vitro diagnostics (IVD) that until now could be certified in risk class $A$ and $B$ based on a conformity assessment by their manufacturer.

- There is no exemption for previously certified IVD.

The common responsibility of NB, EMA \& health technology assessment bodies for consistent decision will require multistakeholder cooperation

- NB will assess the CDx, EMA will assess the benefit risk balance of the medicine, both NB and EMA regulators will need to consider the impact of the CDx on the treatment of patients, in other words, potential changes in the benefit-risk balance of the medicine, and health technology assessment will look at the cost-effectiveness of the combination of CDx and medicine. Cooperation will be indispensable for consistent decision in patients' interest.

CDx may impact the benefit-risk balance \& license status of medicines

- For one medicine, certification of several CDx with different features is possible.

- The benefit-risk balance of the medicine may depend on the performance (e.g., sensitivity and specificity) of a CDx. The use of a different CDx may result in a different benefit-risk balance of the medicine. Inappropriate CDx may ultimately result in regulatory action against the medicine.

Future perspective

- EMA will need to provide detailed information (accuracy, precision, sensitivity and specificity as appropriate) on $C D x$ used in the pivotal trials that were the basis for the positive benefit-risk assessment. Only then other stakeholders, for example, health technology assessment bodies, payers, physicians and patients can build on EMA's assessment and evaluate the suitability of alternate CDx.

- Exceeding the mere description of the characteristics of the CDx used in the pivotal studies, it could help to minimize potentially divergent decisions by NB if EMA defined in their initial assessment of a new medicine the minimum performance of $C D x$ necessary for a positive benefit-risk balance of the medicine. 
teristics of the CDx and the corresponding medicine. Payers may decide whether better accuracy justifies a higher price or a lower accuracy will exclude the product from reimbursement. Physicians and patients may decide on an individual level how much uncertainty they are willing to accept.

A close cooperation and a scientific alignment of EMA, NB and HTA will assure adequate performance standards of certified $\mathrm{CDx}$ and provide favorable conditions for the introduction of competing $\mathrm{CDx}$ products.

\section{Acknowledgements}

The authors greatly appreciate the assistance and contributions of I Bachmann.

\section{References}

Papers of special note have been highlighted as:

- of interest; $\bullet$ of considerable interest

1 Eichler H-G, Hurts H, Broich K, Rasi G. Drug regulation and pricing - can regulators influence affordability? N. Engl. J. Med. 374(19), 1807-1809 (2016).

2 Enzmann H. New trends and challenges in the European regulation of innovative medicines. Regul. Toxicol. Pharmacol. 80, 314-320 (2016).

3 Akhmetov I, Ramaswamy R, Akhmetov I, Thimmaraju P. Market access advancements and challenges in "drugcompanion diagnostic test" co-development in Europe. J. Pers. Med. 5(2), 213-228 (2015).

4 JØrgensen JT. Companion diagnostics in oncology - current status and future aspects. Oncology 85(1), 59-68 (2013).

5 Lee EY, Shen H-CJ. Regulatory considerations for companion diagnostic devices. Biomark. Med. 9(1), 67-75 (2015).

- Describes the US position on companion diagnostics (CDx).

6 Tazawa Y. Perspective for the development of companion diagnostics and regulatory landscape to encourage personalized medicine in Japan. Breast Cancer 23(1), 19-23 (2016).

- Describes the Japanese view of CDx.

7 Council of the EU. Medical devices: deal reached on new EU rules - Consilium. Press release (2016). www.consilium.europa.eu/

8 Pignatti F, Ehmann F, Hemmings R et al. Cancer drug development and the evolving regulatory framework for companion diagnostics in the European Union. Clin. Cancer Res. 20(6), 1458-1468 (2014).

\section{Financial \& competing interests disclosure}

The authors have no relevant affiliations or financial involvement with any organization or entity with a financial interest in or financial conflict with the subject matter or materials discussed in the manuscript. This includes employment, consultancies, honoraria, stock ownership or options, expert testimony, grants or patents received or pending, or royalties.

No writing assistance was utilized in the production of this manuscript.

\section{Open access}

This work is licensed under the Attribution-NonCommercialNoDerivatives 4.0 Unported License. To view a copy of this license, visit http://creativecommons.org/licenses/by-nc-nd/4.0/

- Describes the view of European regulators based on earlier (and different) drafts of the regulation.

9 EMA. Cerdelga. EPAR (2016). www.ema.europa.eu/

10 The European Parliament and the Council of the European Union. Proposal for a regulation of the European Parliament and of the Council on in vitro diagnostic medical devices. (2015).

http://eur-lex.europa.eu/

- The latest version of the proposed regulation. Changes in the wording, for example, of the CDx definition in comparison with the previous version, are perceptible.

11 Carvajal-Hausdorf DE, Schalper KA, Neumeister VM, Rimm DL. Quantitative measurement of cancer tissue biomarkers in the lab and in the clinic. Lab. Investig. 95(4), 385-396 (2015).

12 Bornholz B, Benninghaus T, Reinke Y et al. A standardised FACS assay based on native, receptor transfected cells for the clinical diagnosis and monitoring of $\beta 1$-adrenergic receptor autoantibodies in human heart disease. Clin. Chem. Lab. Med. 54(4), 683-691 (2016).

13 Brandwood A. Brandwood Biomedical. Brexit and the CE mark - implications for European regulation of medical devices (2016). http://brandwoodbiomedical.com/

14 EMA (2016). Herceptin, INN-trastuzumab. EPAR. www.ema.europa.eu/

15 Zaric GS. Cost implications of value-based pricing for companion diagnostic tests in precision medicine. Pharmacoeconomics 34(7), 635-644 (2016).

16 Halim A-B. The biggest challenges currently facing companion diagnostic advancement. Expert Rev. Mol. Diagn. 14(1), 27-35 (2014). 